\title{
Validation of the radiation pattern of the VHF MST radar MAARSY by scattering off a sounding rocket's payload
}

\author{
T. Renkwitz, C. Schult, R. Latteck, and G. Stober \\ Leibniz-Institute of Atmospheric Physics at the Rostock University, Schloss-Str. 6, 18225 Kühlungsborn, Germany \\ Correspondence to: T. Renkwitz (renkwitz@iap-kborn.de)
}

Received: 16 January 2015 - Revised: 14 April 2015 - Accepted: 2 June 2015 - Published: 3 November 2015

\begin{abstract}
The Middle Atmosphere Alomar Radar System (MAARSY) is a monostatic radar with an active phased array antenna designed for studies of phenomena in the mesosphere and lower thermosphere. Its design, in particular the flexible beam forming and steering capability, makes it a powerful instrument to perform observations with high angular and temporal resolution. For the configuration and analysis of experiments carried out with the radar it is essential to have knowledge of the actual radiation pattern. Therefore, during the time since the radar was put into operation various active and passive experiments have been performed to gain knowledge of the radiation pattern. With these experiments the beam pointing accuracy, the beam width and phase distribution of the antenna array were investigated. Here, the use of a sounding rocket and its payload as a radar target is described which was launched in the proximity of the radar. The analysis of these observations allows the detailed investigation of the two-way radiation pattern for different antenna array sizes and beam pointing positions.
\end{abstract}

\section{Introduction}

The Middle Atmosphere Alomar Radar System (MAARSY) was built in 2009/2010 by the Leibniz-Institute of Atmospheric Physics (IAP) on the island Andøya in Northern Norway $\left(69.30^{\circ} \mathrm{N}, 16.04^{\circ} \mathrm{E}\right)$. The MAARSY radar facilitates studies of the arctic atmosphere with high spatial and temporal resolution within the mesosphere/lower thermosphere and the troposphere/lower stratosphere and is therefore categorized as a MST radar. The main active phased array antenna consists of 433 individual 3-element Yagi antennas (see Fig. 1) optimized for approximately $5 \mathrm{MHz}$ bandwidth at the operating frequency of $53.5 \mathrm{MHz}$. Each individual antenna of the main array is connected to its own transceiver allowing independent phase and amplitude settings. This arrangement facilitates very flexible beam forming and steering properties of the radar. A detailed technical description of the radar is given by Latteck et al. (2012). Furthermore, additional external antenna groups or individual antennas may be connected to the receivers allowing e.g. interferometric observations with antenna spacings and baseline lengths divergent to the main array and its subarrays. Extensive simulations of the radiation pattern of the main antenna array and the additional groups have been performed during the design phase and since the radar was put into operation. These simulations were performed with the Numerical Electromagnetic Code (version 4.1) investigating the radiation properties like the antenna gain, beam width and shape, the side lobe suppression, the influence of the soil properties and mutual coupling for various beam pointing directions.

In the beginning passive experiment like the observation of cosmic radio emissions were used to validate the simulation results. By the observation of galactic radio emissions the beam pointing accuracy, the beam width as well as a rough estimation of the side lobe attenuation (Renkwitz et al., 2012, 2013) and the antenna-receiver phase distribution were investigated (Chau et al., 2014). Therein, the passive observations of cosmic radio sources were compared to a highly accurate reference model by de Oliveira-Costa et al. (2008). Additional active two-way radar experiments with MAARSY using targets like satellites, Earth's moon as well as the piecewise comparison to meteor head echo events, already described in the previously given references, augmented the findings. However, the observation of targets within or beyond the Earth's ionosphere are challenging using linearly polarized antennas and needs to be carefully analyzed. Another method of validating the one-way radiation pattern of 


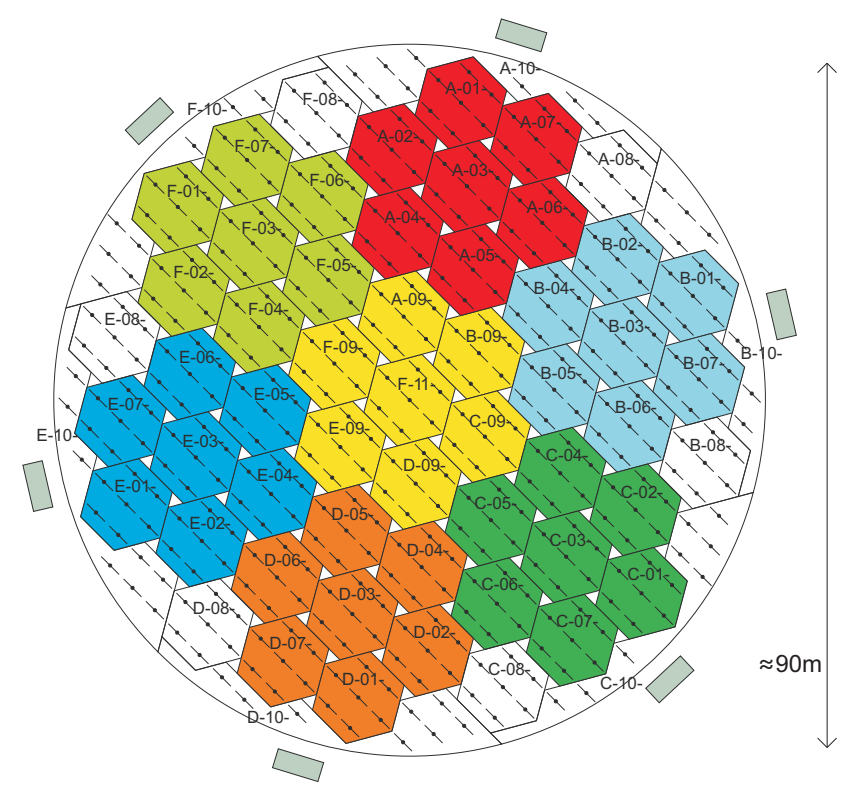

Figure 1. Sketch of the MAARSY VHF radar antenna array with the nomenclature of the individual subarrays. The colored subgroups mark the MAARSY343 subarray.

a VHF antenna array is the placement of e.g. a transmitter onboard a satellite. This has to be carried out carefully as the wave's propagation through the ionosphere is subject to Faraday rotation and is therefore often limited to circularly polarized antenna arrays (see e.g. Fukao et al., 1985; Sato et al., 1989). Alternatively, a receiver may be placed on an airplane or helicopter to sample directly the radar's emissions, advantageously in the radiation far field.

In this paper we present an active radar experiment performed with MAARSY, which allows the determination of the beam pointing accuracy, the effective beam width and side lobe attenuation. For this purpose we use the backscatter of a sounding rocket's payload that was launched in the proximity of the radar. In the subsequent section we will describe the experiment we conducted to evaluate the radiation pattern of the MAARSY radar by the use of the sounding rocket's payload as a radar target. Subsequently we will present and discuss the results of this experiment and compare these to the simulation findings. Finally, conclusions and an outlook will be presented.

\section{Experiment description}

In the end of June 2013 the WADIS sounding rocket campaign (WAve propagation and DISsipation in the middle atmosphere) was conducted at the Andøya Space Center located in the proximity of MAARSY. During this campaign several meteorological and one instrumented rocket were launched. These rocket launches were accompanied by simultaneous operations of various ground based radar, lidar
Table 1. Experiment settings used to observe the WADIS payload.

\begin{tabular}{ll}
\hline Pulse repetition frequency & $1200 \mathrm{~Hz}$ \\
Sampling range & $\approx 75$ to $119 \mathrm{~km}$ \\
Code & 16 bit complementary \\
Pulse length & $2 \mu \mathrm{s}$ \\
\hline Maximum peak power & nom. $433 \times 2 \mathrm{~kW}$ \\
\hline Number of beams & 18 \\
\hline
\end{tabular}

and balloon facilities. Herein, MAARSY was the leading radar facility to detect and observe polar mesospheric summer echoes (PMSE) with multiple beam directions to propose favorable launch conditions and to derive detailed information about the PMSE structure during the flight. For this purpose MAARSY was operated with a dedicated experiment configuration during the flight to sequentially observe 16 different beam directions along the predicted payload's trajectory.

Besides the initial objectives of this scan configuration, the subsequent analysis of the spatially and temporally spread PMSE and the in-situ observations of the rocket's payload, additional backscatter was observed. The analysis of the radar data set turned out to contain strong backscatter from the rocket, the payload and likely the rocket's nose cone. Favorably, the rocket motor and the payload had a length of approximately half the radar wavelength allowing strong backscatter. The beam positions MAARSY was sequentially scanning using the entire antenna array for transmission and the GPS trajectory ${ }^{1}$ of the payload with color-coded height are depicted in the left panel of Fig. 2. The GPS trajectory of the payload has been validated by interferometric means of MAARSY, using various combinations of subarrays carefully calibrated in range and phase. Due to the large radar cross-section of the payload we detected signatures of it in basically all beam positions. Thus, within 75 and $119 \mathrm{~km}$ range the flight of the payload has been observed almost continuously as shown in an power integrated plot in the right panel of Fig. 2. The prominent data gaps are caused by the selected experiment configuration, mainly the chosen sample rate and the available data transfer speed of the acquisition system existent at that time. The experiment settings are shown in Table 1.

The partially strong backscatter of at most $50 \mathrm{~dB}$ signal-tonoise ratio allows the detection of the radar's main lobe and its first side lobe and is therefore exceptionally valuable for radiation pattern comparisons. In the subsequently presented analysis of the experiment's data different sizes of the antennas array are used for reception, where the use of the entire antenna array (MAARSY433) should provide the maximum gain of all configurations in the selected beam pointing direction and therefore the minimum available beam width. Addi-

\footnotetext{
${ }^{1}$ Courtesy of M. Hörschgen-Eggers/DLR-MORABA.
} 

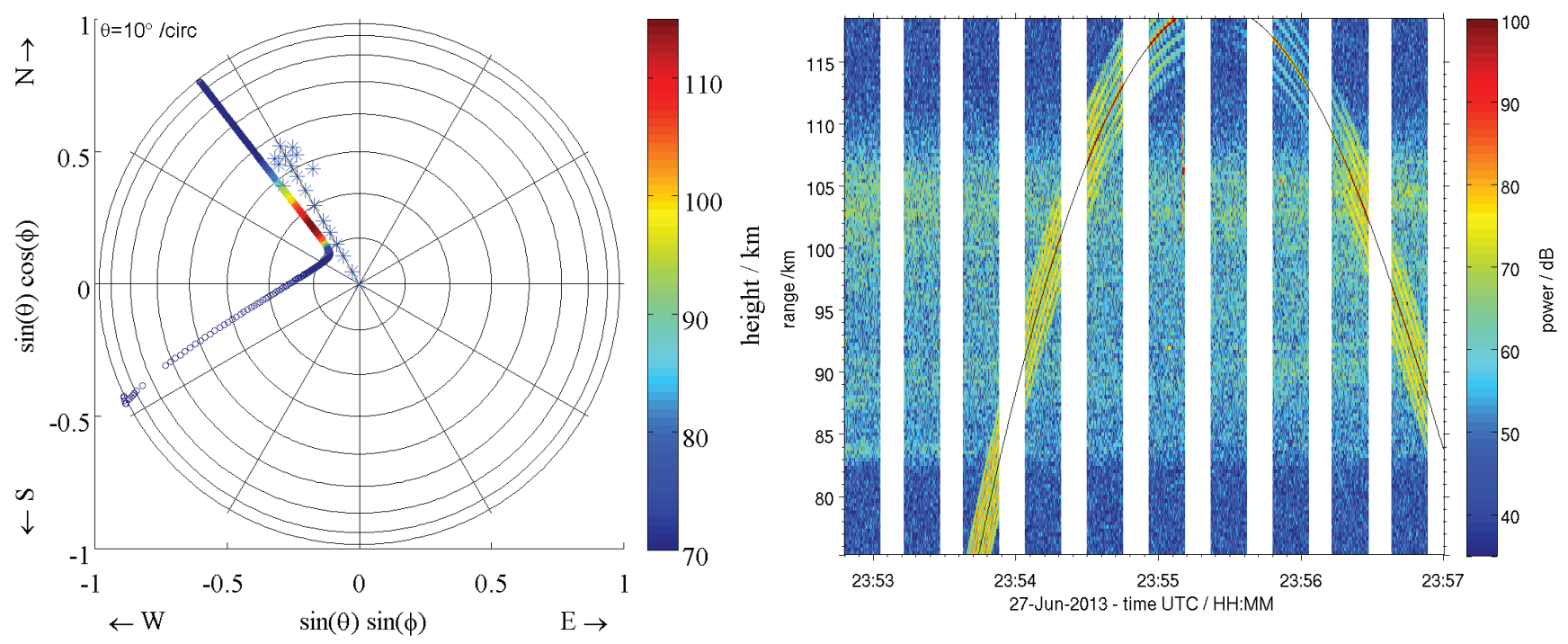

Figure 2. Left panel: trajectory of the WADIS rocket payload in reference to MAARSY, depicted in spherical coordinates, overlaid by the beam positions used in the WADIS scan experiment, marked by asterisks. The height of the payload during its flight is depicted color coded. Right panel: integrated power of all beams in the scan (median removed) depicted over range and time. The thin black line marks the range calculated from the GPS coordinates, which agrees exceptionally well to the observed range during the entire flight. The enhanced intensity between approximately 82 and $106 \mathrm{~km}$ range origins PMSE observed with different beam positions.

tional to this completely hardware combined channel, 15 additional receiving channels have been connected to subarray groups of 49 or 7 antennas each (Anemones and Hexagons, respectively). With the knowledge of the absolute phases of these individual subarray groups larger subarrays may be formed in software by integrating the signals of e.g. seven Anemones resulting in MAARSY343 (marked in color in Fig. 1). This software combined subarray is exceptionally interesting due to its large aperture and thus good angular resolution. The use of different sizes of the antenna array allows the verification of the beam pointing accuracy, beam width and side lobe attenuation for multiple beam directions for every single configuration. Furthermore the use of small subarrays in this experiment is exceptionally interesting as those broad radiation patterns do not contribute significantly to the two-way pattern. As an extreme case the use of an omnidirectional antenna for reception result in a two-way pattern that is identical to the pattern of the one-way transmitting antenna as the two-way pattern is the product of both individual radiation patterns.

The radiation pattern of the corresponding subarray groups and the entire antenna array have been simulated for the respective beam pointing positions for the entire hemisphere with $1^{\circ}$ resolution in both azimuth and zenith angle. Within these simulations the antenna array have been modeled with best accuracy of the antenna structure itself and the soil properties. Thus, the most realistic pattern and so the side lobes are taken into account for the subsequent analysis. The resulting two-way pattern for the different receiving subarrays were computed afterwards to obtain simulated intensi- ties along the payload's trajectory. The simulated intensities were then corrected for the changing radar cross-section of the target during its flight.

For this purpose we assume a metallic mainly pure cylindrical surface and a simplified spherical bottom side of appropriate dimensions. The most significant approximation used here refers to both ends of the payload, where various instruments are placed. These instruments represent diverse surface structures significantly smaller than the used wavelength which makes it intricate to derive a comprehensive expression and therefore a half sphere is assumed. This imperfection is, however, relieved as the total effective area is dominated by the payload's cylindrical body. Considering the length of the target of approximately half the radar wavelength Rayleigh scattering appears most appropriate for the purpose of this analysis and the desired accuracy.

Applying these approximations of the geometric shape the radar cross-sections $\sigma_{\mathrm{s}}$ and $\sigma_{\mathrm{b}}$ for the cylindrical side and the bottom surface may be expressed by the approximations given by Fuhs (1983), respectively.

$$
\begin{aligned}
\sigma_{\mathrm{s}}^{\mathrm{o}} & =9 / 4 \pi h^{2}(2 \pi r / \lambda)^{4} \\
\sigma_{\mathrm{s}}^{\mathrm{p}} & =\frac{\pi \cdot h^{2}}{(\pi / 2)^{2}+(\ln (\lambda / 1.78 \pi r))^{2}} \\
\sigma_{\mathrm{s}} & =\left(\sigma_{\mathrm{s}}^{\mathrm{o}}+\sigma_{\mathrm{s}}^{\mathrm{p}}\right) / 2 \cdot \sin \theta_{\mathrm{a}} \\
\sigma_{\mathrm{b}} & =7.11 \pi r^{2} \cdot(2 \pi / \lambda)^{4} \cdot \cos \theta_{\mathrm{a}}
\end{aligned}
$$

The height and radius of the payload are denoted by $h$ and $r$, which are $2.8 \mathrm{~m}$ and $0.35 \mathrm{~m}$ respectively. The angle of attack from the radar to the payload is represented by $\theta_{\mathrm{a}}$, 


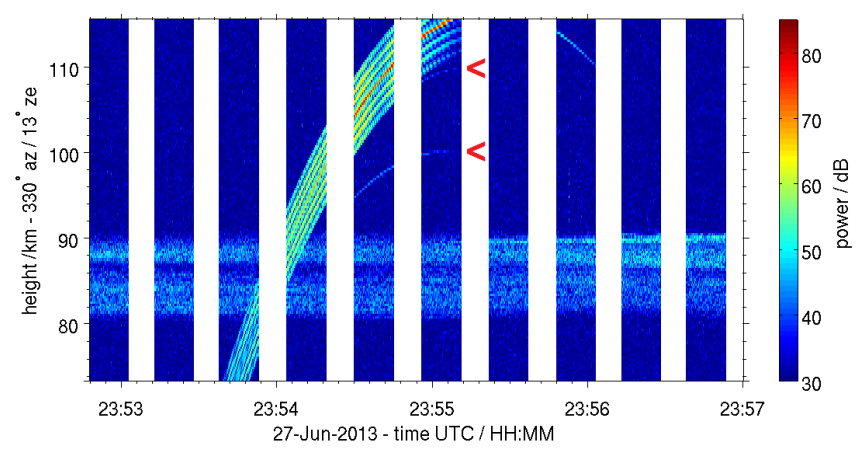

Figure 3. Detected intensities for the beam direction $\phi=330^{\circ}$ and $\theta=13^{\circ}$ using MAARSY433 on reception. The backscatter at around $85 \mathrm{~km}$ height originates PMSE. The red angles mark backscatter likely caused by the motor and the rocket's nose cone.

which varies between 10 to $30^{\circ}$ during the evaluated period. In Eqs. (1) to (3) $\sigma_{\mathrm{s}}^{\mathrm{o}}$ and $\sigma_{\mathrm{s}}^{\mathrm{p}}$ describe the orthogonal and parallel components of the total radar cross section $\sigma_{\mathrm{s}}$ of the cylindrical body, where the first dominates significantly. As the MAARSY antenna array was mainly circularly polarized during the experiments, no consideration of the orientation angle of the payload and the polarization angle are necessary as it would have been for linear polarization near the resonance scattering. Besides this angular independency of circular polarization for the mentioned target it, however, leads to a reduction of effective area by a factor of 2 (in Eq. 3), comparable to the polarization loss between linear and circular polarization. Alternatively, equations and approximations given by e.g. van Vleck et al. (1947), Mailloux (1994) and Skolnik (2008) yield to results of equivalent cross sections. It has to be noted, diffraction and creeping waves around the backside of the body are neglected in the shown approximations. The radar cross-section was computed for every point of the trajectory considering the pitch angle of the rocket (and its payload) and the geometric angle of attack from the radar to the target. The radar cross-section thus varies for the evaluated beam direction by a factor of $3 \mathrm{~dB}$ at most accounting for the previously mentioned approximations. This mainly affects the simulated intensity of the outward side lobe and to a lesser extent the simulated beam width. This factor is mainly caused by the variable effective area of the dominating cylindrical surface with the minimum at approximately $80 \mathrm{~km}$ height during the upleg and the maximum during the downleg due to the increasing zenith angle and thus angle of attack and effective area.

\section{Analysis and discussion}

In this section the analysis of the experiment's data for few beam positions and their interpretation are discussed.

Figure 3 shows the detected intensities for the beam pointing direction of $\phi=330^{\circ}$ and $\theta=13^{\circ}$. Here, besides the sig-

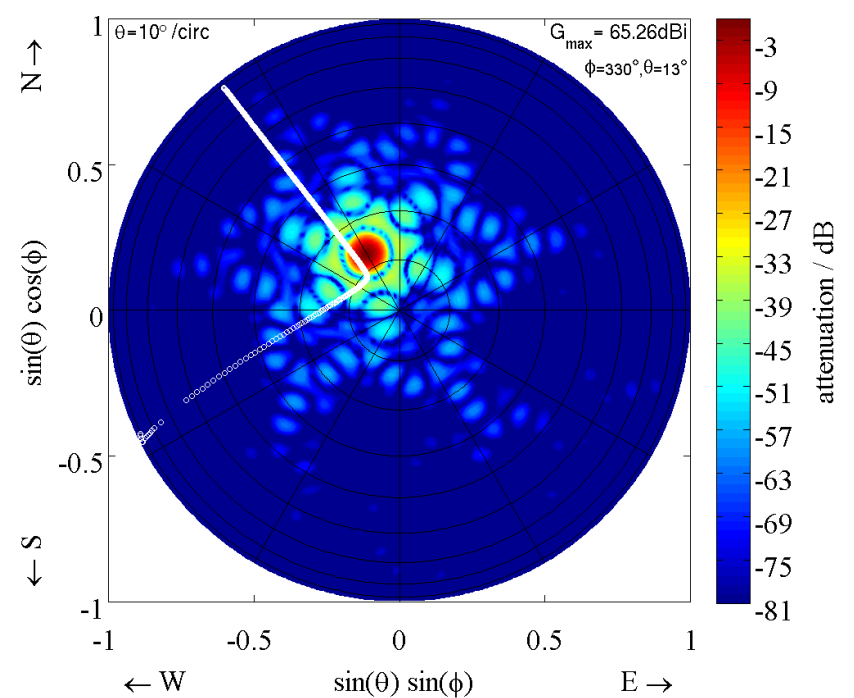

Figure 4. Simulated two-way radiation pattern pointing to $\phi=330^{\circ}$ and $\theta=13^{\circ}$ by the superposition of MAARSY433 and MAARSY343 for transmission and reception, respectively. Equivalently to Fig. 2 left, the trajectory of the payload is marked by white circles.

nature the of PMSE at approximately 81 to $90 \mathrm{~km}$ height, the major backscatter is detected from the rocket's payload almost throughout its maximum range. For the periods of very strong backscatter the observed intensities are marked by the properties of the used code and thus also the code's side lobes smearing $\pm 4 \mathrm{~km}$ around the maximum. Since 23:54 UT the maximum of the payload's detected intensity per time agrees well to the range and height calculated from the GPS trajectory. Prior to 23:54 UT the backscatter intensity does not maximize in the middle of the signature. This indicates imperfect decoding of the signal due to the large Doppler shift caused by the high departure speed in respect to the radar's position. Unfortunately no un-decoded raw data have been stored as the experiment's outcome was not foreseen and thus its potential needs were unknown during the configuration process. Interestingly, below the strong payload backscatter two additional weaker signatures can be spotted at approximately $100 \mathrm{~km}$ and $109 \mathrm{~km}$ apogee respectively, which are most likely caused by the rocket's motor and the nose cone, marked by red angles in Fig. 3. The reason of seeing the payload's backscatter during the entire upleg with this beam position can be seen in Fig. 4, where MAARSY's two-way radiation pattern is shown overlaid by the trajectory of the payload. The payload essentially stays within the main lobe and the first side lobe ring for the beam pointing of $\phi=330^{\circ}$ and $\theta=13^{\circ}$. The beginning downleg can be seen e.g. for the beam pointing of $\phi=330^{\circ}$ and $\theta=20^{\circ}$ (see Fig. 2, respectively). Here, the payload traverses a part of the side lobe ring of MAARSY and thus allows short but strong backscatter ( $40 \mathrm{~dB}$ signal-to-noise ratio). Furthermore, only weak traces can be seen during the upleg period as for this beam direction 


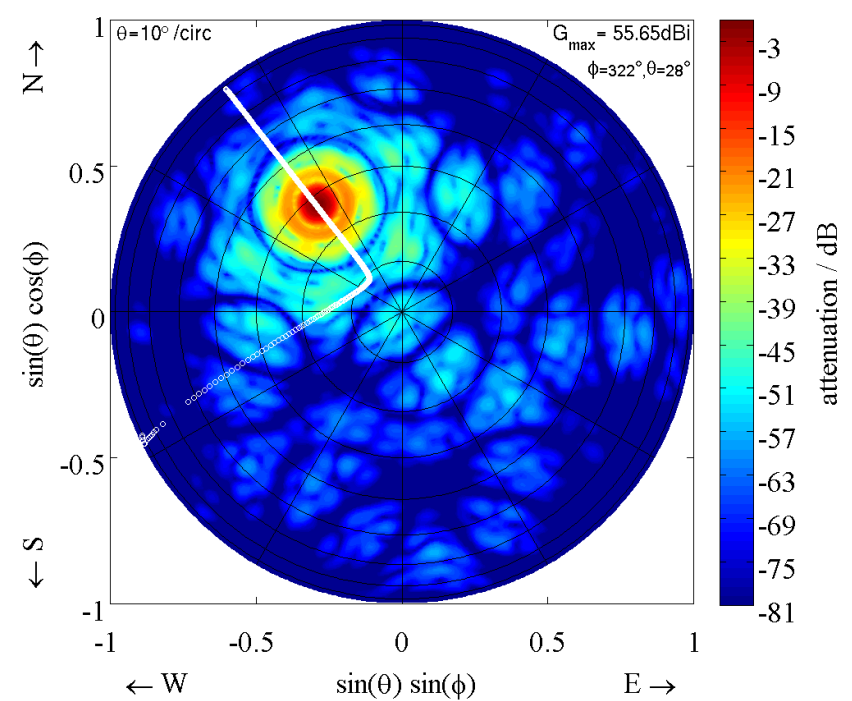

Figure 5. Simulated two-way radiation pattern pointing to $\phi=322^{\circ}$ and $\theta=28^{\circ}$ by the superposition of MAARSY433 and an Anemone group of 49 antennas for transmission and reception, respectively. Equivalently to Fig. 2 left, the trajectory of the payload is marked by white circles.

the payload was far outside the main and the first side lobe resulting in at least $40 \mathrm{~dB}$ less gain in these directions. More interesting are the observations for the earlier highlighted beam pointing direction of $\phi=322^{\circ}$ and $\theta=28^{\circ}$ (see Fig. 5).

Here, the payload almost exactly passes through the radar's beam pointing direction and thus facilitates strong backscatter from both, the main lobe and the first side lobe ring (see Fig. 6). Therefore this beam pointing direction was used for the detailed analysis and comparison with the simulated radiation pattern. For this purpose the maximum detected intensities close to the GPS trajectory are used, as marked with crosses in Fig. 7 for both MAARSY433 (blue) and MAARSY343 (red) on reception. The simulated intensities, computed for the actual state of the antenna array during this experiment for reception and transmission, are depicted as solid lines in the respective color for both array sizes.

Similarly to the detected power in Fig. 6, the intensities have been simulated for each point of the GPS trajectory and thus the direction from the radar to the target. It can be seen that the relative intensities and the beam shape for both array sizes are approximately the same. MAARSY's beam pointing appears to be shifted very slightly towards zenith, which is however hardly to see due to the existing data gap at the simulated maximum intensity. Nevertheless the misalignment can be approximated to be in the order of less than $0.5^{\circ}$. The observed beam width for MAARSY433 on reception appears to be slightly broader than the width of MAARSY343. This is just opposite to the simulation and the general approximation since the aperture of the MAARSY433 supersedes MAARSY343 and thus the theoretical relative beam width is inversely proportional to their aperture ratio. Furthermore,

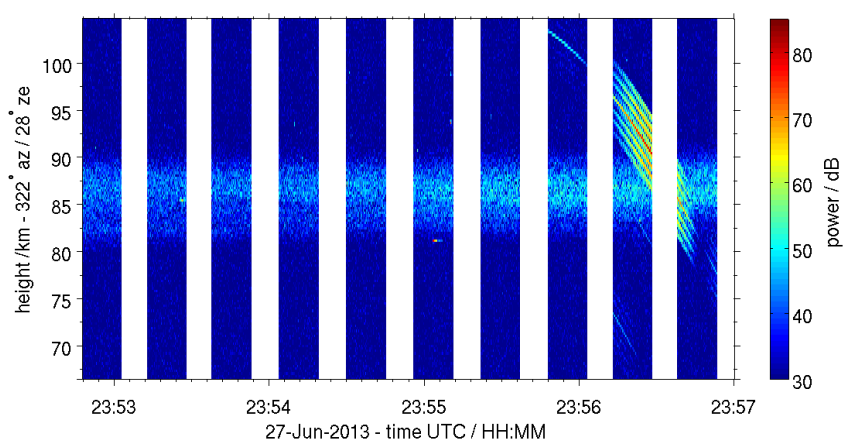

Figure 6. Detected intensities for the beam direction $\phi=322^{\circ}$ and $\theta=28^{\circ}$ using MAARSY343 on reception. The backscatter at around $85 \mathrm{~km}$ height originates PMSE.

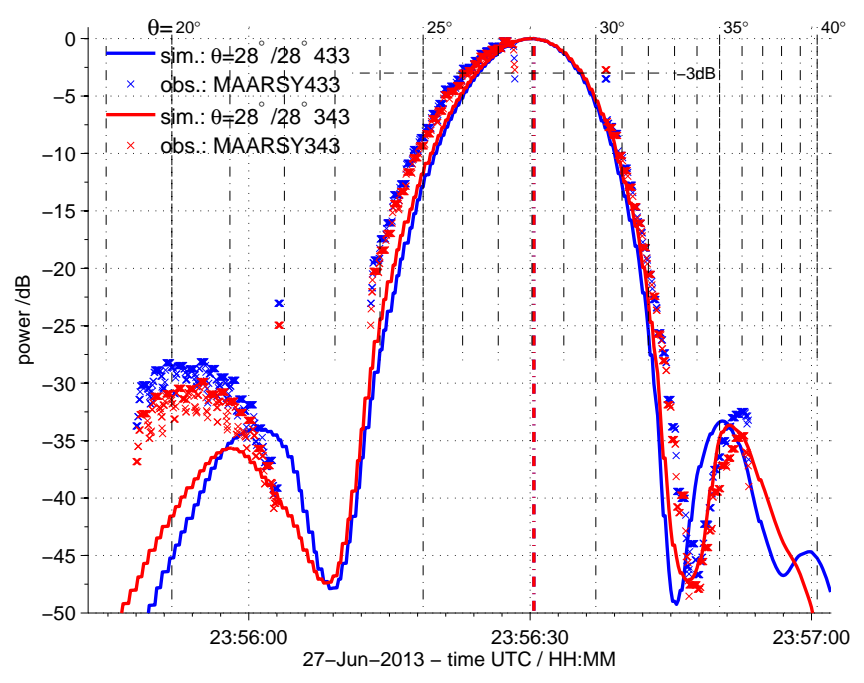

Figure 7. Detected and simulated intensities (marked by crosses and solid line, respectively) for the beam direction $\phi=322^{\circ}$ and $\theta=28^{\circ}$ using MAARSY433 and MAARSY343 (blue and red, respectively). Black vertical broken lines mark the zenith angle labeled on top of the figure. Colored vertical broken line accentuates the maximum of simulated intensity.

the beam width of the main lobe of both arrays seem to be broadened by $0.5^{\circ}$ at most, which is consistent to the outward shifted positions of the minima between the main lobe and the first side lobe ring. The side lobe attenuation agrees fairly well to the simulations with about $2 \mathrm{~dB}$ increased intensity towards zenith.

It has to be noted that the MAARSY antenna array was mixed-polarized during this experiment. Since May 2013 the antennas of the seven Anemones (A to F and the Middle, see Fig. 1) were completely converted from linear to circular polarization, while the outer antennas were still linearly polarized. Additionally, during that time in total 21 transmitter modules and thus antennas were non-operational on transmission, which disturbs the radiation pattern mainly affect- 


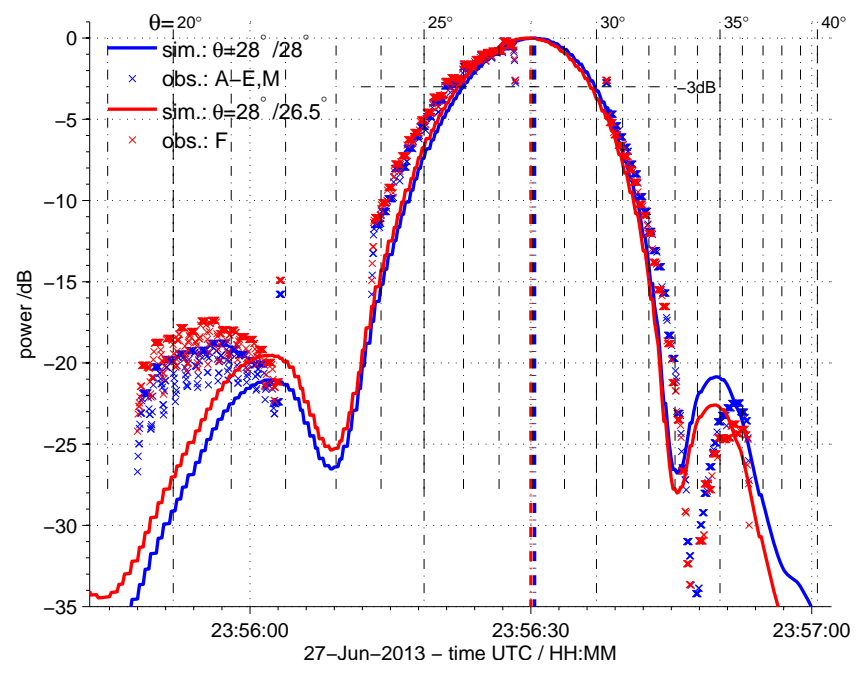

Figure 8. Equivalent to Fig. 7. Detected intensities for the beam direction $\phi=322^{\circ}$ and $\theta=28^{\circ}$ using Anemones A-E, M (blue) and Anemone $\mathrm{F}$ (red) marked by crosses. The corresponding simulated intensities are shown for $\phi=322^{\circ}$ and $\theta=28^{\circ}$ (blue) and $\phi=322^{\circ}$ and $\theta=26.5^{\circ}$ (red).

ing the side lobe level. Both issues have already been incorporated in the simulations used for this comparison.

To investigate the reason of the broadened beam width smaller subarray groups have been used in a similar manner as before. In Fig. 8 the simulated and observed intensities for Anemone subarrays (49 antennas each) are shown. It was found that six out of the seven available Anemones show equivalent detected intensities, while Anemone F slightly differed. The main lobe of Anemone F seems to be shifted towards zenith, which is also underlined by an intensified side lobe towards zenith and vice versa with the outer side lobe. The best agreement to the observations was found for the simulation of pointing the Anemone at most $1.5^{\circ}$ off the nominal beam direction. This misalignment however should not result in broadening the beam width of the entire antenna array in such an extent as seen before. Equivalent to the previous example using (almost) the entire antenna array, the two-way beam width using the Anemone subarrays on reception appears to be broadened by $0.5^{\circ}$. Consistently, the minima between main and side lobe are shifted outwards by approximately $0.6^{\circ}$.

As quoted in the prior section a rather broad, in an extreme case omnidirectional, antenna does not change the two-way radiation pattern significantly. During this experiment, the smallest available antenna groups were Hexagon subarrays, composed of seven antennas each. In total eight Hexagons spread over the antenna array have been used and analyzed (see Fig. 9). Besides mediocre variation of the detected intensities via the side lobes (e.g. B-08), all Hexagons show an equivalent shape and width of the main and side lobe. However, the beam width appears to be still enlarged by approx-

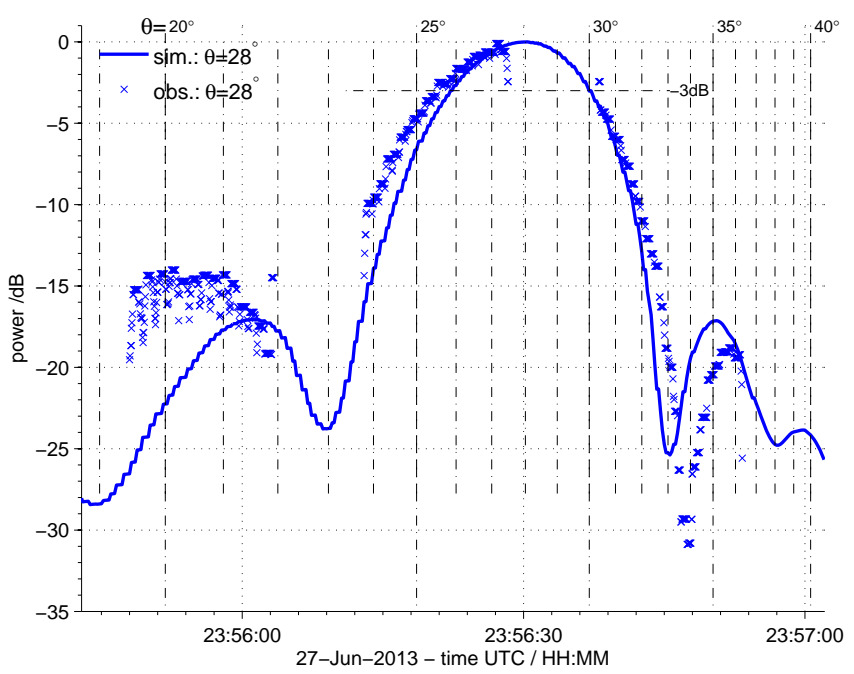

Figure 9. Detected intensities for the beam direction $\phi=330^{\circ}$ and $\theta=13^{\circ}$ using a Hexagon group of seven antennas.

imately $0.7^{\circ}$. Due to the wide beam width of the Hexagons $\left(31^{\circ}\right)$ the effective width of the two-way radiation pattern is dominated by the transmission pattern of the entire antenna array. Even though the width of the Hexagons could also be enlarged to some extent it appears to be more likely the beam width of the entire antenna array is broadened.

A generally smaller aperture of the antenna array and thus broader beam width as planned can be excluded as the array has been built with best precision. Furthermore passive experiments observing cosmic radio sources and active experiments observing meteor head echoes indicated a better agreement with simulations (see e.g. Renkwitz et al., 2012, 2013; Chau et al., 2014).

Possible reasons of the observed broadening are: (a) additional ineffective antennas at the rim of the entire antenna array, (b) unintentional amplitude taper, or (c) imperfect simulation. The outermost antennas of the entire array have not been exceptionally peculiar in neither earlier experiments nor the automatically measured impedances and reflected output power for each experiment run for the considered time. Unintentional random amplitude taper $( \pm 1 \mathrm{~dB})$ and phase variations $\left( \pm 10^{\circ}\right)$ for the individual antennas have been simulated and are shown in Fig. 10. The perfectly phased array MAARSY343 with uniform amplitude has a minimum beam width of $4^{\circ}$, while for the simulation with random phase and amplitude variations a broadening of $0.1^{\circ}$ can be seen in one cross-section. An imperfect simulation may be caused by the mixed polarization within the antenna array, leading to e.g. divergent mutual coupling, or the non-operational transmitter modules and thus individual antennas (21 in total). Simulations of the entire antenna array considering these missing antennas have shown a beam width broadening approximately of $0.2^{\circ}$, which already has been incorporated in the simulations of the previously shown comparisons to the 

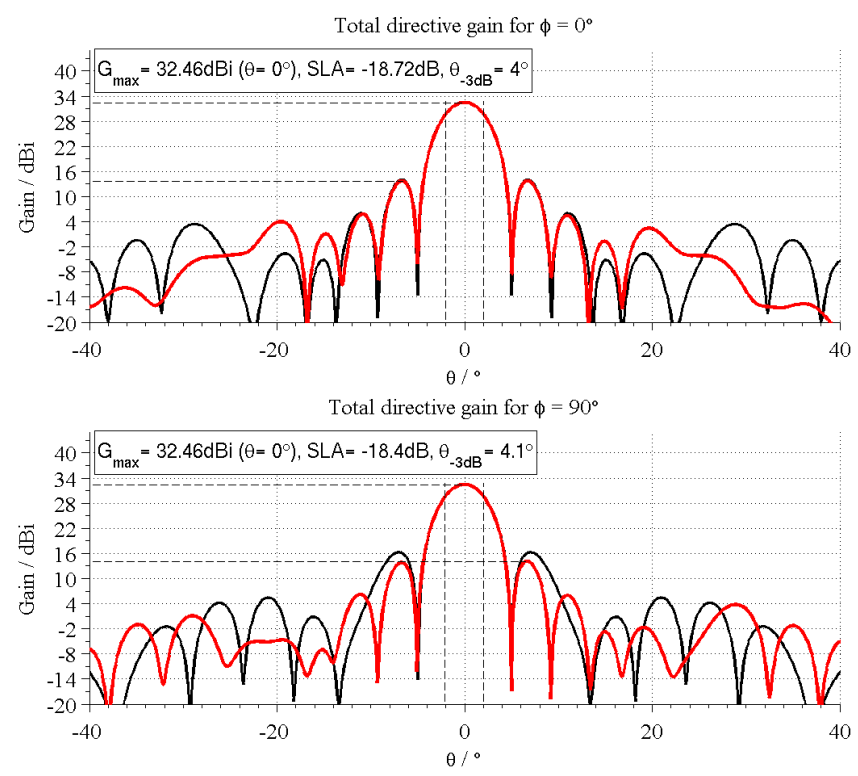

Figure 10. Comparison of simulations for the ideally phased and uniform amplitude MAARSY343 (black) to the case of random fluctuations of every array element of $\pm 1 \mathrm{~dB}$ and $\pm 10^{\circ}$ for two cross-sections $\left(\phi=0^{\circ}\right.$ and $\left.\phi=90^{\circ}\right)$.

observations. Finally, we assume a superposition of primarily the points (b) and (c) causing the observed beam width broadening of approximately $0.5^{\circ}$.

\section{Conclusions and outlook}

In this paper we presented an active experiment to validate the radiation pattern of MAARSY. This actively scanning radar experiment was initially planned to derive background parameters, like PMSE strength and wind components for the trajectory of the rocket's payload launched during the WADIS campaign. Additionally to these objectives strong backscatter from the payload were detected which facilitates the comparison to the simulated two-way radiation pattern of the radar. During this experiment the payload's flight could be seen in all 18 scheduled beam directions along the predicted trajectory. This is primarily caused by the payload's size (almost exactly half wave length of the radar) and its cylindrical shape that exhibit a large radar crosssection. Though MAARSY reaches a reasonably good side lobe suppression, the payload's backscatter could often also be seen in the first side lobe of the radar's radiation pattern for the entire antenna array and smaller subarrays on reception. The two-way radiation pattern for the combination of the entire antenna array on transmission and various arrays sizes for reception were simulated for the individual points of the payload's trajectory. These simulations were subsequently corrected for the varying radar cross-section due to the orientation of the payload and thus the angle of attack.
Doing so, we were able to find very good agreement of the simulated radiation pattern and the observed backscatter intensities. Though, we also found a generally enlarged beam width, which could be verified by the use of smaller subarrays on reception, seven antennas at the least. Therefore the transmit pattern incorporating the entire antenna array already has to be broadened. The enlarged beam width is most likely caused by the superposition of circularly polarization emitted by approximately $80 \%$ of the antenna array in its center and the still linearly polarized antennas at the rim of the array. Furthermore, with the simulation we could demonstrate that the subarray Anemone $\mathrm{F}$ appeared to squint to an extent of maximum $1.5^{\circ}$ towards zenith during this experiment. This is assumed to be caused by a flaw in the receiver phases, which however could not be verified during maintenance measures. Overall, we are impressed by the excellent opportunity to validate the radiation pattern by the observation of a sounding rocket, which is of significant benefit to the radar operators. Therefore, we are looking forward to the next equivalent rocket campaign in the proximity of the radar as MAARSY's antenna array is completely converted to circular polarization since autumn 2013. Such rocket campaign could be used to validate the beam width of MAARSY's radiation pattern for the entirely circularly polarized antenna array. For the first half-year of 2015 a second rocket launch within the WADIS campaign is now planned with an equivalent payload. The authors are looking forward to this excellent opportunity which may solve some of the uncertainties illustrated here.

Acknowledgements. The authors explicitly acknowledge the kindly provided GPS trajectory by M. Hörschgen-Eggers/DLR-MORABA and the PI of IAP's sounding rocket group Dr. Boris Strelnikov. The WADIS project was funded by the German Space Agency (DLR) under grant $50 \mathrm{OE}$ 1001. Furthermore, we like to express our gratitude to the Andøya Space Center for their permanent support for the operation and maintenance of the MAARSY radar. The radar development was supported by the german grant 01 LP 0802A of Bundesministerium für Bildung und Forschung.

Edited by: R. Schuhmann

Reviewed by: two anonymous referees

\section{References}

Chau, J. L., Renkwitz, T., Stober, G., and Latteck, R.: MAARSY multiple receiver phase calibration using radio sources, J. Atmos. Sol.-Terr. Phy., doi:10.1016/j.jastp.2013.04.004, 2014.

de Oliveira-Costa, A., Tegmark, M., Gaensler, B. M., Jonas, J., Landecker, T. L., and Reich, P.: A Model of Diffuse Galactic Radio Emission from $10 \mathrm{MHz}$ to $100 \mathrm{GHz}$, Mon. Not. Roy. Astron. Soc., 338, 247-260, doi:10.1111/j.13652966.2008.13376.x, 2008.

Fuhs, A. E.: Radar cross section lectures, Naval postgraduate school Monterey, Dep. of Aeronautics, California, 1983. 
Fukao, S., Sato, T., and Kato, S.: Monitoring of the MU Radar Antenna Pattern by Satellite OHZORA (EXOS-C), J. Geomag. Geoelectr., 37, 431-441, 1985.

Latteck, R., Singer, W., Rapp, M., Vandepeer, B., Renkwitz, T., Zecha, M., and Stober, G.: The new MST radar on Andøya: System description and first results, Radio Science, 47, RS1006, doi:10.1029/2011RS004775, 2012.

Mailloux, R. J.: Phased Array Antenna Handbook, vol. 1, 1st Edn., Artech House, London, 1994.

Renkwitz, T., Singer, W., Latteck, R., Stober, G., and Rapp, M.: Validation of the radiation pattern of the Middle Atmosphere Alomar Radar System (MAARSY), Adv. Radio Sci., 10, 245-253, doi:10.5194/ars-10-245-2012, 2012.
Renkwitz, T., Stober, G., Latteck, R., Singer, W., and Rapp: New experiments to validate the radiation pattern of the Middle Atmosphere Alomar Radar System (MAARSY), Adv. Radio Sci., 11, 283-289, doi:10.5194/ars-11-283-2013, 2013.

Sato, T., Inooka, Y., Fukao, S., and Kato, S.: Multi-Beam Pattern Measurement of the MU Radar Antenna by Satellite OHZORA, J. Geomag. Geoelectr., 41, 743-752, 1989.

Skolnik, M. I.: Radar handbook, 3rd Edn., McGraw-Hill, New York, 2008.

van Vleck, J. H., Bloch, F., and Hamermesh, M.: Theory of radar reflection from wires or thin metallic stripes, J. Appl. Phys., 18, 274-294, doi:10.1063/1.1697649, 1947. 